\title{
LY 294002 inhibits adenosine receptor activation by a mechanism independent of effects on PI-3 kinase or casein kinase II
}

\author{
T.J. Searl \& E.M. Silinsky \\ Department of Molecular Pharmacology and Biological Chemistry (MPBC), Northwestern University Medical School, \\ Chicago, Illinois, USA
}

Received 13 April 2005; accepted in revised form 12 July 2005

Key words: acetylcholine, adenosine, casein kinase, neuromuscular junction, neurotransmitter release, PI-3 kinase

\begin{abstract}
Adenosine reduces both evoked and spontaneous calcium-dependent acetylcholine (ACh) release through a mechanism downstream of calcium entry at amphibian motor nerve endings (Silinsky EM. J Physiol 1984; 346: 243-56). LY 294002 (2-(4-morpholinyl)-8-phenyl-4H-1-benzopyran-4-one), an inhibitor of both phosphoinositide-3 kinase (PI-3 kinase) and casein kinase II, has been reported to increase spontaneous ACh release reflected in miniature endplate potential (MEPP) frequencies independently of intraterminal calcium at the frog neuromuscular junction (Rizzoli SO, Betz WJ. J Neurosci 2002; 22: 10680-9). It has been suggested that the increase in MEPP frequency caused by LY 294002, is mediated through an action on synaptotagmins, vesicle associated calcium sensors believed to trigger synaptic vesicle exocytosis. We thus examined the effects of adenosine on MEPP frequencies and evoked ACh release reflected as endplate potentials (EPPs) in order to determine if the presumed calcium-independent ACh release is affected by adenosine. We also wanted to determine if PI-3 kinase or casein kinase II is involved in mediating or modulating the inhibitory effects of adenosine. To these ends, we examined the effects of adenosine in the presence of LY 294002, wortmannin (a highly selective the PI-3 kinase inhibitor), or DRB (5,6-dichlorobenzimidazole riboside, an inhibitor of casein kinase II). LY 294002 reduced the sensitivity of both MEPP frequencies and the nerve-evoked calcium dependent EPPs to adenosine. The occlusive effects of LY 294002 on the actions of adenosine on MEPPs and EPPs were overcome by increasing adenosine concentration. Neither wortmannin nor DRB had any effect on the sensitivity of the EPPs to adenosine indicating that neither PI-3 kinase nor casein kinase II inhibition mediates the reduction in motor-nerve terminal sensitivity to adenosine produced by LY 294002. The results indicate a competitive relationship between LY 294002 and adenosine at $\mathrm{A}_{1}$ receptors at the frog neuromuscular junction. This effect is independent of the previously described effects of LY 294002 on the exocytotic process, and is also independent of PI-3 kinase or casein kinase II.
\end{abstract}

Abbreviations: ACh - acetylcholine; DRB - 5,6-dichlorobenzimidazole riboside; EPPs - end-plate potentials; LY 294002 2-(4-morpholinyl)-8-phenyl-4H-1-benzopyran-4-one; MEPPs - miniature end-plate potentials; PI-3 kinase-phosphoinositide-3 kinase

\section{Introduction}

Activation of $A_{1}$ receptors by adenosine is known to inhibit the release of acetylcholine (ACh) from frog motor nerve terminals through a mechanism that occurs downstream of calcium entry [1-4]. In the frog, the effects of the activation of $A_{1}$ receptors by adenosine on both the spontaneous MEPPs and nerve-evoked EPPs are comparable, with the maximal inhibition for both classes of events

\footnotetext{
Correspondence to: Dr E.M. Silinsky Department of Molecular Pharmacology and Biological Chemistry (MPBC), Northwestern University Medical School, 303 East Chicago Avenue, Chicago, IL 60611, USA Tel: +1-312-5038287; Fax: +1-312-5030796; E-mail: e-silinsky@ northwestern.edu
}

being in the order of $50 \%[2,5,6]$. The rapid timing and stimulus independence of this inhibition indicates that adenosine must act on vesicles which are in a post-primed fully release-competent state $[7,8]$. Thus, the most likely candidates for mediating the inhibition of transmitter release by $A_{1}$ receptor activation at the frog neuromuscular junction are the core components of the exocytosis machinery i.e., the SNARE proteins (SNAP-25, syntaxin and synaptobrevin), the calcium sensor synaptotagmin or a thus far uncharacterised element of the secretory apparatus. On the basis of biophysical evidence it was previously suggested that inhibition of ACh release by adenosine occurs through an action on the calcium sensor responsible for triggering release $[1,7]$. More recently we found further evidence for this suggestion [9] using the compound 4-aminoquinaldine. In brief we found that, at the 
frog neuromuscular junction, application of 4-aminoquinaldine induces the appearance of a population of calcium insensitive giant size MEPPs, quantal synaptic events that are independent of a functional calcium sensor [9]. In addition to the lack of sensitivity to calcium, these events are not affected by adenosine, leading us to conclude that a functional form of synaptotagmin is necessary for mediating the actions of adenosine on transmitter release at the frog neuromuscular junction.

Given the apparent importance of synaptotagmin in mediating the inhibition of release of acetylcholine by adenosine, and of PI-3 kinase and casein kinase in modulating the secretory apparatus [10], it seems possible that agents that alter synaptotagmin function or these two kinases might also affect the action of adenosine at motor nerve endings. Work by Rizzoli and Betz [10] suggests that 2(4-morpholinyl)-8-phenyl-4H-1-Benzopyran-4-one (LY 294002) an inhibitor of both phosphoinositide-3 kinase and casein kinase II is a promising candidate for these experiments. As reported by these coworkers, application of LY 294002 causes a substantial acceleration of MEPP frequency at the frog neuromuscular junction, through a mechanism that is independent of intraterminal calcium [10]. As a result Rizzoli and Betz proposed that LY 294002 causes the release of MEPPs through a perturbation of synaptotagmin function.

The aim of this work was thus twofold. Firstly, we wished to determine whether MEPPs released as a result of this proposed perturbation of synaptotagmin function by LY 294002 are subject to the same regulation by adenosine as normal calcium-dependent release at the frog neuromuscular junction. Secondly we wished to determine if any effects of LY 294002 on the action of adenosine is mediated by PI-3-kinase or casein kinase II.

\section{Materials and methods}

\section{General}

Frogs (Rana pipiens) were killed by anaesthesia with $5 \%$ ether, followed by double pithing, in accordance with guidelines laid down by our institutional animal welfare committee. Isolated cutaneous-pectoris nerve-muscle preparations were used in all experiments. Intracellular recordings were made using microelectrodes filled with $3 \mathrm{M} \mathrm{KCl}$ with resistances 3-10 $\mathrm{M} \Omega$. The signal from the microelectrode was fed into a conventional high-input impedance microelectrode pre-amplifier (Axoclamp-2A, Axon Instruments), the signal was then passed from the axoclamp through an $\mathrm{AC}$ coupled filter with a $10 \mathrm{KHz}$ low pass filter. Responses were fed into a personal computer using a Digidata 1200 (Axon Instruments) and digitized at a $0.1 \mathrm{~ms}$ sampling rate. Solutions were delivered by superfusion with a peristaltic pump and removed by vacuum suction. In the experiments on the effects of adenosine and 2-chloroadenosine on EPPs recorded in the presence of either LY 294002 or wortmannin, EPPs were evoked at $0.02 \mathrm{~Hz}$, in order to minimize the rate of EPP run-down. In addition, solution exchange was maximized with a $5 \mathrm{ml} / \mathrm{min}$ superfusion rate of the $1.5 \mathrm{ml}$ recording chamber. Lastly, in order to have the maximal accuracy for these experimental results, a maximum of three concentrations of adenosine was tested between washes in the LY 294002 experiments. When necessary, corrections to the EPP amplitudes were made assuming a linear rundown rate (see inset to Figure 3). All experiments were carried out at room temperature $\left(22-24{ }^{\circ} \mathrm{C}\right)$.

Normal recording solutions contained (mM): $\mathrm{NaCl}, 115$; $\mathrm{KCl}, 2$; HEPES, 2; and $\mathrm{CaCl}_{2}, 1.8 \mathrm{mM}$. Records of MEPPs were made (418.5 s recording duration) and analysed using CDR and SCAN programs (DOS versions, Strathclyde University Value-Packed Software; John Dempster). In experiments where the effects of drugs on the endplate potentials (EPPs) was determined, preparations were paralyzed with $10 \mu \mathrm{M}(+)$-tubocurarine. EPPs were evoked by stimulation of the nerve at low frequencies $(0.02 \mathrm{~Hz})$.

\section{Statistical methods}

Data are presented as the mean \pm S.E.M. In most cases, the data groups were first tested for normality and then tested for significance using one-way analysis of variance (Sigma Stat, Jandel Scientific Inc). A Student's $t$-test was then used to compare individual groups. Differences between groups were considered significant when $P<0.05$, but in some cases further statistical details are provided. Unless otherwise stated, $n$ represents the number of single experiments carried out at single end-plates.

Drugs

LY 294002 and wortmannin were purchased from Tocris Bioscience, Ellisville, USA. Adenosine, 2-chloroadenosine and DBR were purchased from Sigma St Louis, USA.

\section{Results}

\section{LY 294002-evoked MEPPS and the effects of adenosine}

In our initial experiments we found that the application of LY $294002(150 \mu \mathrm{M})$ produced a 3.5 fold increase in MEPP frequency similar to that previously described by Rizzoli and Betz [10]. Specifically, in control conditions, MEPP frequencies were $0.8 \pm 0.2 \mathrm{~Hz}$; following application of $150 \mu \mathrm{M}$ LY 294002, MEPP frequencies increased to $3.5 \pm 0.9 \mathrm{~Hz}(n=5, P<0.05)$.

We next compared the effects of adenosine on normal MEPPs and on MEPPs evoked by LY 294002. In control experiments, $25 \mu \mathrm{M}$ adenosine produced a $51+3.8 \%$ $(n=4)$ reduction in MEPPs. This is a similar to the maximal inhibition by adenosine previously reported for MEPPs [6]. In contrast, $25 \mu \mathrm{M}$ adenosine had no significant $(P>0.05)$ effect on MEPPs evoked by LY 294002. In Figure 1, which depicts a typical experiemtal result, the control MEPP frequency (Figure 1a) was $3.9 \mathrm{~s}^{-1} \pm 0.1 \mathrm{~s}^{-1}$ $($ mean +1 S.E.M.) and following application of adenosine 
$(25 \mu \mathrm{M})$, MEPP frequency was $3.6 \pm 0.1 \mathrm{~s}^{-1}$ (Figure 1b). Figure 2, which shows the average results from five experiments, also illustrates that the inhibitory effect of LY 294002 was surmountable. Specifically, LY 294002 obtunds the inhibitory effects of adenosine (black bar) when compared to control (open bar) yet increasing the concentration of adenosine to $200 \mu \mathrm{M}$ produced the same level of inhibition of LY 294002-induced MEPPs (grey bars) as that produced by $25 \mu \mathrm{M}$ adenosine on normal MEPPs (for further statistical details, see Figure legend). These results support the suggestion that alteration of synaptotagmin function by LY 294002 might reduce the potency of adenosine as an inhibitor of spontaneous ACh release at the frog neuromuscular junction.

\section{The effect of LY 294002 on the inhibition of EPPS by adenosine}

In order to investigate whether the failure of $25 \mu \mathrm{M}$ adenosine to decrease MEPP frequency relates to the calcium independence of LY 294002-evoked MEPPs, experiments were then carried out on the effects of adenosine on evoked ACh release (i.e., EPPs) in the presence and absence of LY 294002. If the reduced potency of adenosine is due to the calcium insensitive of the LY 294002 MEPPs, then the $25 \mu \mathrm{M}$ adenosine should have its usual inhibitory effect on EPPs in the presence of LY294002 as evoked ACh release is calcium-dependent.

As previously found [10], LY 294002 caused an immediate initial increase in EPP amplitudes followed by a slower incremental reduction in EPP amplitudes (see Figure 3). The reduction in EPP amplitudes is attributable to the known inhibition by LY 294002 of PI-3 kinase (an enzyme which is known to play a crucial role in recycling vesicles for re-release - see [10-12]). Our adenosine a

LY 294002

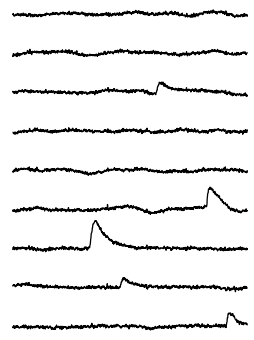

b

LY $294002+$ $25 \mu \mathrm{M}$ adenosine

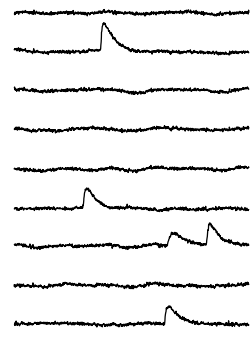

$2 \mathrm{mV}$
Figure 1. The effect of adenosine $(25 \mu \mathrm{M})$ is greatly obtunded in the presence of LY $294002(150 \mu \mathrm{M})$. (A) shows control MEPPs generated by LY 294002 and (B) shows the effects of adenosine on these MEPPs. MEPP frequency in LY 294002 alone was $3.9 \mathrm{~s}^{-1} \pm 0.1 \mathrm{~s}^{-1}$ (mean +1 S.E.M.), following application of adenosine $(25 \mu \mathrm{M})$, MEPP frequency was $3.6 \pm 0.1 \mathrm{~s}^{-1}$ (B). This is in contrast to the approximately $50 \%$ inhibition produced by adenosine in the absence of LY 294002 [2]. The membrane potential in this experiment was $-67 \mathrm{mV}$ throughout.

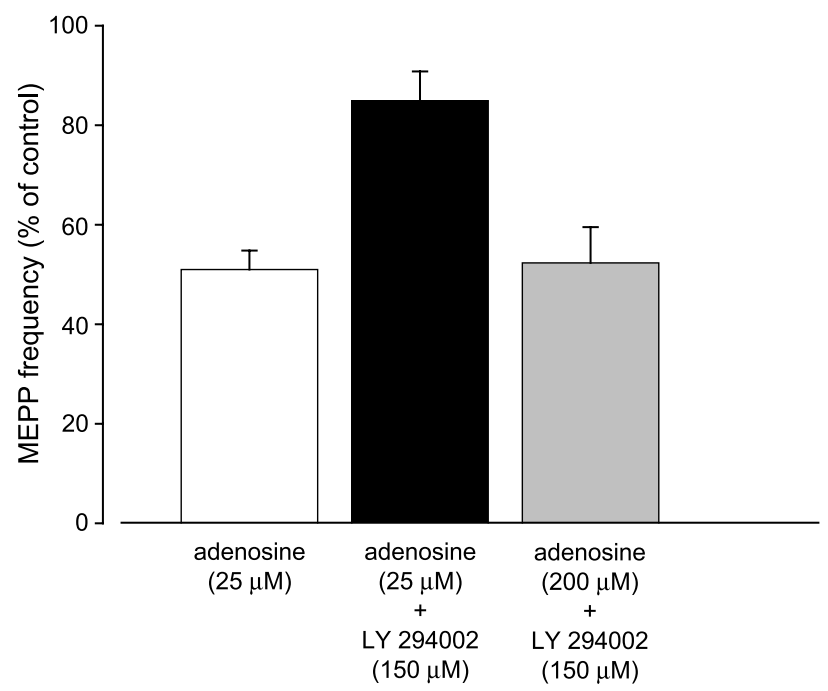

Figure 2. The effect of adenosine on MEPP frequencies recorded in the absence and the presence of $150 \mu \mathrm{M}$ LY 294002. In the absence of LY 294002, $25 \mu \mathrm{M}$ adenosine reduced MEPP frequencies to $51 \pm 3.8 \%$; $n=4,(P<0.05)$ of that recorded in the absence of adenosine (open bar; $100 \%=1.1 \mathrm{~Hz}$ ). In the presence of $150 \mu \mathrm{M}$ LY 294002 MEPP frequencies were not significantly reduced by $25 \mu \mathrm{M}$ adenosine $(84.9 \pm$ $5.9 \% ; n=5$, of that recorded in the absence of adenosine, black bars; $\left.100 \%=2.7 \mathrm{~s}^{-1}\right)$. The effect was surmountable by increasing the concentration of adenosine to $200 \mu \mathrm{M}-52.3 \pm 7.2 \% ; n=5,(P<0.05$, grey bar $; 100 \%=4.3 \mathrm{~s}^{-1}$ ).

concentration response experiments were thus carried out towards the end of this second phase in the action of LY 294002, where the EPP amplitudes are only declining very slowly (see inset to Figure 3 ). In order to minimize the rate of run-down, EPPs were evoked at $0.02 \mathrm{~Hz}$. As shown in Figure 4a, adenosine also had a reduced potency and efficacy on EPPs in the presence of LY $294002(150 \mu \mathrm{M}$, filled squares) when compared with control (open circles). It thus appears that calcium-dependent and calcium independent ACh release both have the same reduced sensitivity to adenosine in the presence of LY 294002.

\section{The effect of LY 294002 on the inhibition of EPPS by 2-chloroadenosine}

It could be argued that the reduction in the potency of adenosine might be a result of modifications in the activities of nucleoside transporters, adenosine deaminase, adenosine kinase, or another mechanism involved in the elimination of adenosine. While this suggestion seemed unlikely, given the degree of the reduction in the potency of adenosine seen, we could not entirely rule it out. The selective $A_{1}$ receptor agonist 2-chloroadenosine is not a substrate for either deamination or uptake [2]. We therefore tested the effects of $150 \mu \mathrm{M}$ LY 294002 on the action of 2-chloroadenosine with a partial dose response curve. As shown in Figure 4b, LY 294002 produced a reduction in the potency of 2-chloroadenosine (filled squares) from the control level (open circles) that is comparable to the reduction in the potency of adenosine (Figure $4 a$ ). 


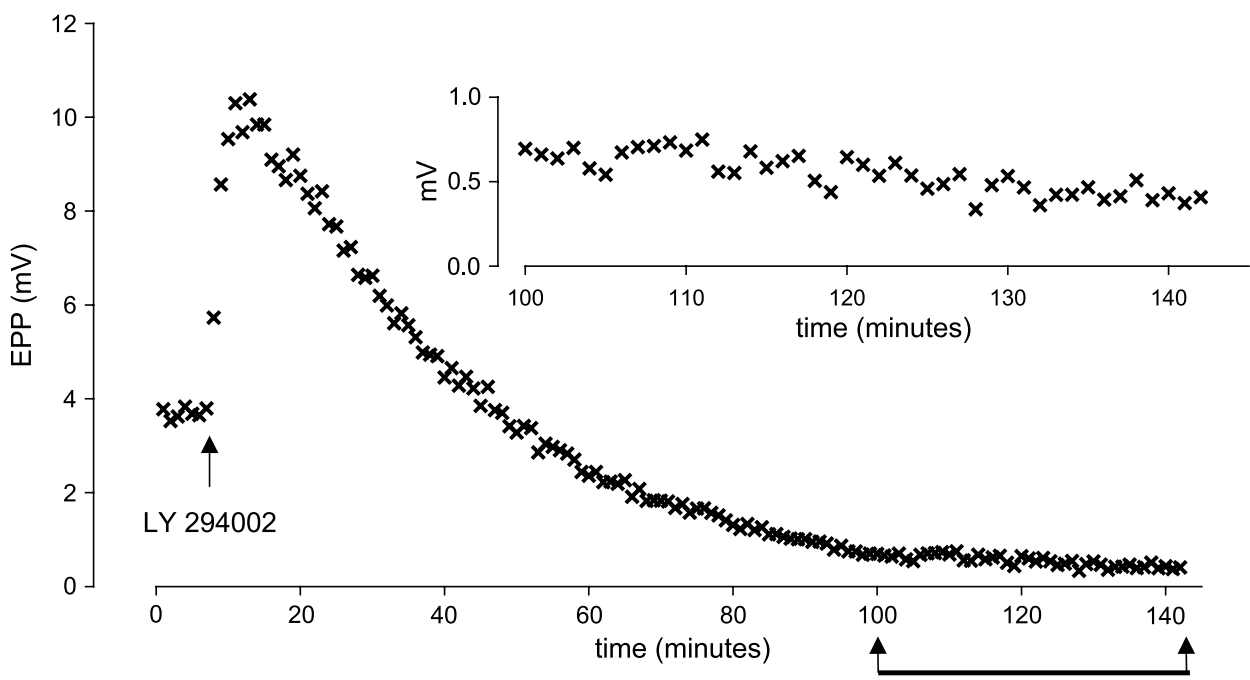

Figure 3. The effect of LY294002 $(150 \mu \mathrm{M})$ on EPP amplitudes. The graph shown illustrates the effects of LY 294002 on EPPs in a single representative cell. Each point plotted represents a single evoked EPP. As shown, following application of LY $294002(150 \mu \mathrm{M})$, EPPs initially increased in amplitude. After approximately 10 min following the initial application of LY 294002 EPPs began to rundown. Following 90 min in the presence of LY 294002 the rate in rundown in EPP amplitudes was reduced such that the effects of adenosine could be tested (see arrows and inset). The membrane potential was $-103 \mathrm{mV}$ throughout the course of this experiment.

The similar inhibition by LY 294002 of the action of adenosine on presumed synaptogtagmin-independent MEPPs and on calcium and synaptotagmin-dependent EPPs suggests that the LY 294002-adenosine interaction is unrelated to a selec- tive perturbation of synaptotagmins. As LY 294002 inhibits both PI-3 kinase and casein kinase II, we next wished to determine if the reduction in sensitivity of the release process to adenosine was due to inhibition of either of these two enzymes.

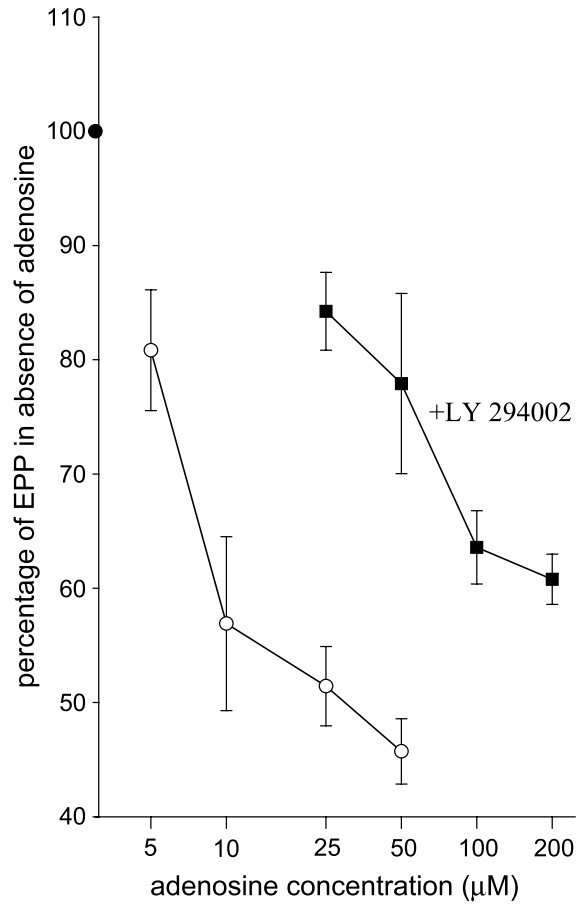

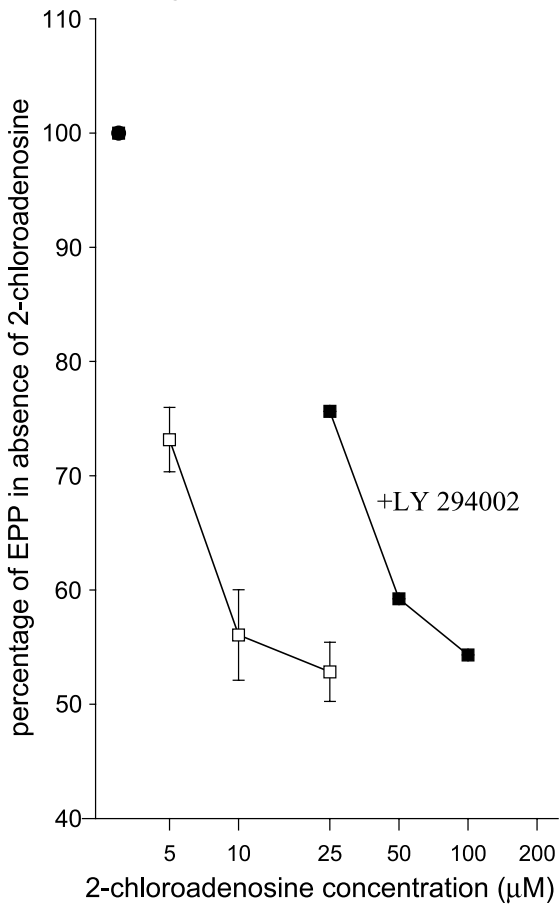

Figure 4. The effect of the LY $294002(150 \mu \mathrm{M})$ on the concentration-dependent inhibition of EPP amplitudes by adenosine (a) and 2-chloroadenosine (b). As shown in (a), the potency of adenosine was significantly reduced by the presence of $150 \mu \mathrm{M}$ LY 294002 . In control the concentration of adenosine that produced a $50 \%$ inhibition of ACh release was $6.7+1.8 \mu \mathrm{M} n=4$, in LY 294002 this concentration was $59.0+21.4 \mu \mathrm{M}(n=4)$. As shown in (b), the potency of 2-chloroadenosine was significantly reduced by the presence of $150 \mu \mathrm{M} L \mathrm{Y} 294002$. In control, threshold the concentration of 2-chloroadensoine that reduced ACh release to $50 \%$ of the control level was $3.9+0.5 \mu \mathrm{M} n=4$, in LY 294002 this value was $32.0+11.8 \mu \mathrm{M}(n=4$, $P<0.05$ ). These results suggest that the reduction in the potency of adenosine produced by LY 294002 at the frog neuromuscular junction is not mediated by the upregulation of the endogenous mechanisms responsible for the elimination/degradation of adenosine by LY 294002 . Each point on the concentration response curves represents the average \pm SEM of $n=4$ experiments. 


\section{Is PI-3 kinase inhibition or casein kinase II inhibition responsible for the reduced potency of adenosine?}

We first investigated the effects of the PI-3 kinase inhibitor wortmannin $(3 \mu \mathrm{M})$ on the adenosine dose response curve. As previously described [11, 12], $3 \mu \mathrm{M}$ wortmannin produced a gradual stimulus dependent decline in EPP amplitudes. This effect has been attributed to an impairment of the PI-3 kinase dependent recycling process. Inhibition of recycling is maximal with a concentration of $1 \mu \mathrm{M}$ wortmannin [12]. However, the effectiveness of adenosine as an inhibitor of evoked release was unchanged (see Figure 5, filled circles).

We then investigated the effects of the casein kinase II inhibitor $300 \mu \mathrm{M}$ DRB [13] on the inhibition of $\mathrm{ACh}$ release by adenosine. This concentration of DRB has been previously shown to mimic the increase in MEPPs produced by LY 294002 [10]. It was previously shown that $300 \mu \mathrm{M}$ DRB produced a five fold increase in MEPP frequency [10] which is comparable with the 3-4 fold increase in MEPPs we found with $150 \mu \mathrm{M}$ LY 294002. However, as shown in Figure 5 (filled triangles), $300 \mu \mathrm{M}$ DRB had no effect on the inhibition of EPPs by adenosine. These results suggest that the reduced potency of adenosine in the presence of LY 294002 is not the result of either

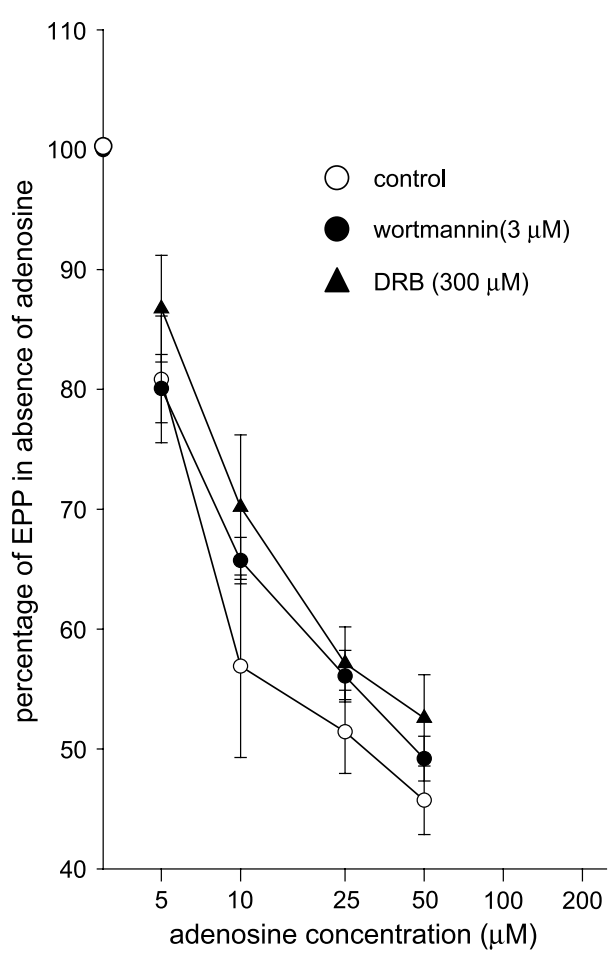

Figure 5. The effect of the PI-3 kinase inhibitor wortmannin $(3 \mu \mathrm{M})$ and the casein kinase II inhibitor DRB $(300 \mu \mathrm{M})$ on the concentration dependent inhibition of EPP amplitudes by adenosine. As shown, neither wortmannin nor DRB had any significant effect on the adenosine concentration dependent inhibition of EPP amplitudes. Each point on the concentration respose curves represents the average \pm SEM of $n=4$ experiments. The threshold concentration of adenosine that inhibited $\mathrm{ACh}$ release to $50 \%$ of the control level in control experiments was $6.5 \pm 1.9$ $\mu \mathrm{M}(n=4)$. In the presence of wortmannin this value was $6.7 \pm 0.4 \mu \mathrm{M}(n$ $=4)$ and in the presence of DRB $(300 \mu \mathrm{M}) 9.4+1.9(n=4) ;(P>0.05)$ inhibition of PI-3 kinase or inhibition of casein kinase II, but rather through an action on adenosine receptor signalling cascade.

\section{Discussion}

In this study, we confirmed previous findings that the application of LY $294002(150 \mu \mathrm{M})$ produced an increase in MEPP frequencies. In addition, we found that there was a reduced sensitivity of the motor nerve ending to adenosine in the presence of this compound. This reduction in sensitivity to adenosine was demonstrated in both the effects of adenosine on MEPP frequencies and on evoked EPPs and hence was not due to any selective effect of LY 294002 to generate calcium independent $\mathrm{ACh}$ release through an interaction with casein kinase II [10]. Rather the results suggest an apparent competitive relationship between $\mathrm{A}_{1}$ adenosine receptor activation and LY 294002. This effect is unrelated to inhibition of PI-3 kinase or casein kinase II as neither the selective PI-3 kinase inhibitor, wortmannin, nor the casein kinase inhibitor II, DRB, had any effect on the sensitivity of nerve evoked EPPs to adenosine.

LY 294002 has been found to have a number of different actions in a variety of systems. As well as its inhibitory actions on PI-3 kinase and casein kinase II [14], it has been reported to inhibit both DNA dependent kinase [15] and the mammalian target of rapamycin (mTOR) [16]. In addition, LY 294002 has been shown to increase intracellular calcium in both bovine and human airway smooth muscle [17]. With respect to interactions between LY 294002 and adenosine, it has previously been reported in the hamster vas deferens smooth muscle cell line $\mathrm{DDT}_{1} \mathrm{MF}-2$, that LY $294002(100 \mu \mathrm{M})$ blocks the phosphorylation of protein kinase $\mathrm{B}$ mediated by activation of the $A_{1}$ receptors [18]. In those experiments, wortmannin $(100 \mathrm{nM})$ also blocked the phosphorylation of protein kinase $B$ triggered by the $A_{1}$ receptor agonist $\mathrm{N}^{6}$-cyclopentyladenosine, leading to the suggestion that $A_{1}$ receptors mediate phosphorylation of protein kinase $\mathrm{B}$ through a PI-3 kinase dependent pathway in that system [18]. However, this mechanism does not seem likely at the frog neuromuscular junction as both the speed at which $\mathrm{A}_{1}$ receptor activation inhibits transmitter release $(<100 \mathrm{~ms}$ [19]), and the failure of wortmannin to affect the sensitivity of evoked EPPs to adenosine, appears to preclude a role for PI-3 kinase as the mechanism by which adenosine inhibits transmitter release in this preparation.

It might be argued that the lack of effect of wortmannin on the adenosine concentration response curve might be due to a lower effectiveness of wortmannin against PI-3 kinase. However, the concentration of wortmannin we used exceeds the concentration of wortmannin $(30 \mathrm{nM})$ used in the studies on the $\mathrm{DDT}_{1} \mathrm{MF}-2$ cells. In addition, wortmannin produced a stimulation dependent decline in EPP amplitude, an effect consistent with inhibition of PI-3 kinase $[13,14]$. Thus, it seems unlikely that inhibition of PI-3 kinase is responsible for the reduction in sensitivity to adenosine produced by LY 294002 in this study. Similarly, 
the failure of the casein kinase inhibitor II, DRB, to alter the sensitivity of adenosine actions on EPPs as is evidenced by the lack of effect on the adenosine concentration response curves, makes it unlikely that inhibition of casein kinase II activity by LY 294002 underlies the decrease in effectiveness of adenosine. However, we acknowledge that we did not directly measure the activity of either kinase in these studies so we cannot entirely rule out a role for either kinase in reducing the sensitivity of the motor nerve to adenosine. The observation that the reduction in sensitivity of the frog neuromuscular junction to adenosine can be overcome by increasing adenosine concentration (both for evoked release and for the release of the spontaneous MEPPs) is consistent with there existing a competitive relationship between adenosine and LY 294002 at the level of the $A_{1}$ receptor in this preparation. While we cannot exclude an effect of LY 294002 at the level of the Gprotein, pertussis toxin progressively inactivates G-proteins and occludes the inhibitory action of adenosine in a manner that is not surmountable by increasing the concentration of adenosine [20].

In conclusion, LY 294002 reduces the sensitivity of both evoked and spontaneous release of ACh to the actions of adenosine at the frog neuromuscular junction. It seems likely that this action of LY 294002 is not due to its known effects on either PI-3 kinase activity or on casein kinase II nor due to its proposed actions on synaptotagmins, but rather is mediated through a direct action on $\mathrm{A}_{1}$ receptors. As a consequence of these findings, further caution needs to be applied to the use of LY 294002 for studies of PI-3 kinase action, particularly in those systems where $A_{1}$ receptor may play a role in cell signaling.

\section{Acknowledgement}

This work was supported by a Grant from the NIH of the USPHS (NS 12782).

\section{References}

1. Silinsky EM. On the calcium receptor that mediates depolarizationsecretion coupling at cholinergic motor nerve terminals. $\mathrm{Br} \mathrm{J}$ Pharmacol 1981; 73: 413-29.

2. Silinsky EM. On the mechanism by which adenosine receptor activation inhibits the release of acetylcholine from motor nerve endings. J Physiol 1984; 346: 243-56.
3. Redman RS, Silinsky EM. ATP released together with acetylcholine as the mediator of neuromuscular depression at frog motor nerve endings. J Physiol 1994; 477: 117-27.

4. Robitaille R, Thomas S, Charlton MP. Effects of adenosine on $\mathrm{Ca}^{2+}$ entry in the nerve terminal of the frog neuromuscular junction. Can $\mathrm{J}$ Physiol Pharm 1999; 77: 707-14.

5. Ribeiro JA, Walker J. The effects of adenosine triphosphate and adenosine diphosphate on transmission at the rat and frog neuromuscular junctions. Br J Pharmacol 1975; 54: 213-8

6. Hunt JM, Silinsky EM. Ionomycin-induced acetylcholine release and its inhibition by adenosine at frog motor nerve endings. $\mathrm{Br} \mathrm{J}$ Pharmacol 1993; 110: 828-32.

7. Searl TJ, Silinsky EM. Phorbol esters and adenosine affect the readily releasable neurotransmitter pool by different mechanisms at amphibian motor nerve endings. J Physiol 2003; 553: 445-56.

8. Silinsky EM, Hirsh JK, Searl TJ et al. Quantal ATP release from motor nerve endings and its role in neurally mediated depression. Prog Brain Res 1999; 120: 145-58.

9. Searl TJ, Silinsky EM. Modulation of $\mathrm{Ca}^{2+}$-dependent and $\mathrm{Ca}^{2+}$ independent miniature endplate potentials by phorbol ester and adenosine in frog. Br J Pharmacol 2005; in press (doi:10.1038/sj/ bjp.0706248).

10. Rizzoli SO, Betz WJ. Effects of 2-(4-morpholinyl)-8-phenyl-4H-1benzopyran-4-one on synaptic vesicle cycling at the frog neuromuscular junction. J Neurosci 2002; 22: 10680-9.

11. Cremona O, De Camilli P. Phospoinositides in membrane traffic at the synapse. J Cell Sci 2001; 114: 1041-52.

12. Richards DA, Rizzoli SO, Betz WJ. Effects of wortmannin and latrunculin A on slow endocytosis at the frog neuromuscular junction. J Physiol 2004; 557: 77-91.

13. Meggio F, Shugar D, Pinna LA. Ribofuranosyl-benzimidazole derivatives as inhibitors of casein kinase-2 and casein kinase-1. Eur J Biochem 1990; 187: 89-94.

14. Davies SP, Reddy H, Caivano M, Cohen P. Specificity and mechanism of action of some commonly used protein kinase inhibitors. Biochem J 2000; 351: 95-105.

15. Izzard RA, Jackson SP, Smith GC. Competitive and noncompetitive inhibition of the DNA-dependent kinase. Cancer Res 1999; 59: 2581-6.

16. Brunn GJ, Williams J, Sabers C et al. Direct inhibition of the signaling functions of the mammalian target of rapamycin by the phosphoinositide 3-kinase inhibitors, wortmannin and LY294002. EMBO J 1996; 15: 5256-67.

17. Ethier MF, Madison JM. LY294002, but not wortmannin, increases intracellular calcium and inhibits calcium transients in bovine and human airway smooth muscle cells. Cell Calcium 2002; 32: 31-8.

18. Germack R, Dickenson JM. Activation of protein kinase B by the $\mathrm{A}_{1}$-adenosine receptor in $\mathrm{DDT}_{1} \mathrm{MF}-2$ cells. Br J Pharmacol 2000; 130: $867-74$

19. Silinsky EM, Searl TJ, Redman RS, Hirsh JK. Release and effects of ATP and its derivatives at cholinergic synapses. Drug Dev Res 2001; 52: 22-33.

20. Silinsky EM, Solsona CS, Hirsh JK. Pertussis toxin prevents the inhibitory effects of adenosine and unmasks adenosine-induced excitation at mammalian motor nerve endings. Br J Pharmacol 1989; 97: 16-8. 